\title{
International Journal of RADIATION BIOLOGY
}

Contents: Vol. $59 \quad$ No. $5 \quad$ May 1991

\section{RADIATION CHEMISTRY}

$\mathrm{SO}_{4}^{-}$-induced oxidation of 1,3,6-trimethyluracil and 1,3,5-trimethyluracil (1,3dimethylthymine) by potassium peroxodisulphate in aqueous solution: an interesting contrast

R. Rashid, F. Mark, H.-P. Schuchmann and C. von Sonntag . . . . . . . .

Ultraviolet difference spectral studies in the $\gamma$-radiolysis of DNA and model compounds. II. Oxygen and temperature dependence in adenine radiation chemistry V. M. Stumpo and C. N. Trumbore .

\section{MUTATIONS}

Dependence of the mutation spectrum in a shuttle plasmid replicated in human lymphoblasts on dose of gamma radiation

M. O. Sikpi, M. L. Freedman, E. R. Ziobron, W. B. Upholt and A. G. Lurie

\section{DNA BREAKS}

Kinetics of radiation-induced strand break formation in single-stranded pyrimidine polynucleotides in the presence and absence of oxygen; a time-resolved lightscattering study

G. D. D. Jones and P. O'Neill

\section{CHROMOSOMES}

Reaction kinetics for the development of radiation-induced chromosome aberrations

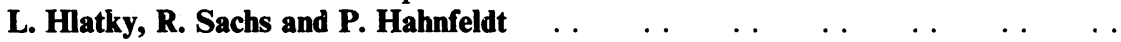

\section{CELL STRUCTURE}

Expression of cytoskeletal elements in proliferating cells following radiation exposure

G. E. Woloschak and C.-M. Chang-Liu $\ldots \quad \ldots \quad \ldots \quad \ldots \quad \ldots \quad \ldots \quad \ldots$

Effect of hyperthermia and lipid peroxidation on the erythrocyte membrane structure

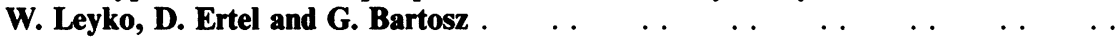

\section{CELL SURVIVAL}

The radiosensitivity of human keratinocytes: influencce of activated $c$ - $H$-ras oncogene expression and tumorigenicity

M. S. Mendonca, P. Boukamp, E. J. Stanbridge and J. L. Redpath . . $\quad \ldots \quad$. 1195

\section{PLD REPAIR}

Repair of potentially lethal damage in rat mammary clonogens following irradiation in organoid culture

K. Kamiya, N. D. Kim, M. N. Gould and K. H. Clifton N $\quad \ldots \quad$. . .

\section{PROTECTION/SENSITIZATION}

Radioprotective properties of DNA methylation-disrupting agents

J. F. Kalinich, G. N. Catravas and S. L. Snyder $\quad \ldots \quad \ldots \quad \ldots \quad \ldots \quad \ldots$

Biochemical effects of heat shock and caffeine on post-irradiation oxic and anoxic damage in barley seeds of low and high water content

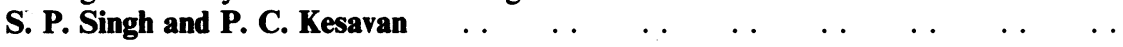

The oxygen effect: variation of the $K$-value and lifetimes of $\mathrm{O}_{2}$-dependent damage in some glutathione-deficient mutants of Escherichia coli

H. A. Harrop, K. D. Held and B. D. Michael 


\section{FRACTIONATION}

A nonparametric method for the derivation of $\alpha / \beta$ ratios from the effect of fractionated irradiations

D. Chmelevsky, R. Guttenberger, J. Kummermehr and A. M. Kellerer

\section{CHELATION}

The efficacy of DFO-HOPO, DTPA-DX and DTPA for enhancing the excretion of plutonium and americium from the rat

G. N. Stradling, S. A. Gray, J. C. Moody, A. Hodgson, K. N. Raymond, P. W. Durbin,

S. J. Rodgers, D. L. White and P. N. Turowski

\section{ABSTRACTS}

Abstracts of the Netherlands Radiobiological Society Working Party 'Clinical Experimental Therapy' of the Dutch Cancer Society

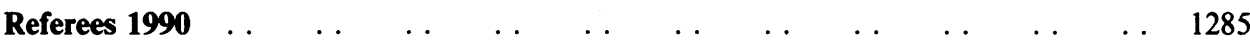

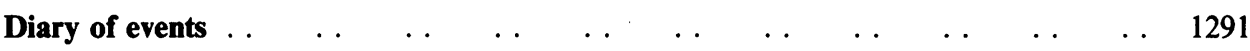

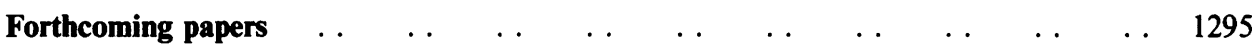

\section{Copyright (C) 1991 Taylor \& Francis Ltd}

All rights reserved. Authors are themselves responsible for obtaining permission to reproduce copyright material from other sources and are required to sign a form for agreement of the transfer of copyright. All requests from third parties to reprint material held in copyright by Taylor \& Francis must be referred to the author for consent as a condition of the granting by Taylor \& Francis of permission for reproduction. No part of this publication may be reproduced, stored in a retrieval system or transmitted in any form, or by any means, electronic, electrostatic, magnetic, mechanical, photocopying, recording or otherwise, without prior permission in writing from the copyright holder.

Authorization to photocopy items for internal or personal use, or the internal or personal use of specific clients, is granted by Taylor \& Francis Ltd for libraries and other users registered with the Copyright Clearance Center (CCC) Transactional Reporting Service, provided that the base fee is paid directly to CCC, 27 Congress Street, Salem, Massachusetts 01970, USA. (For fee per article, see copyright code on first page of each article.) This consent does not extend to other kinds of copying, such as copying for general distribution, for advertising, for creating new collective works, or for resale. Fees for past articles are the same as those shown for current articles. 
INT. J. RADIAT. BIOL., 1991, vol. 59, NO. 5, 1253-1268

\title{
A nonparametric method for the derivation of $\alpha / \beta$ ratios from the effect of fractionated irradiations
}

\author{
D. CHMELEVSKY†, R. GUTTENBERGER $\ddagger$, \\ J. KUMMERMEHR $\ddagger$ and A. M. KELLERER $\ddagger \S$ \\ $\dagger$ Institut für Strahlenschutz der GSF, Ingolstädter Landstr. 1, \\ D-8042 Neuherberg, Germany \\ †Institut für Strahlenbiologie der GSF, Ingolstädter Landstr. 1, \\ D-8042 Neuherberg, Germany \\ $\S$ Strahlenbiologisches Institut der Universität München, Schillerstr. 42, \\ D 8000 München, Germany
}

(Received 6 August 1990; revision received 5 December 1990;
accepted 10 December 1990)

\begin{abstract}
Multifractionation isoeffect data are commonly analysed under the assumption that cell survival determines the observed tissue or tumour response, and that it follows a linear-quadratic dose dependence. The analysis is employed to derive the $\alpha / \beta$ ratios of the linear-quadratic dose dependence, and different methods have been developed for this purpose. A common method uses the so-called $F_{\mathrm{e}}$ plot. A more complex but also more rigorous method has been introduced by Lam et al. (1979). Their method, which is based on numerical optimization procedures, is generalized and somewhat simplified in the present study. Tumour-regrowth data are used to explain the nonparametric procedure which provides $\alpha / \beta$ ratios without the need to postulate analytical expressions for the relationship between cell survival and regrowth delay.
\end{abstract}

\section{Introduction}

Two recent experimental studies (Guttenberger et al. 1990, Kummermehr unpublished) deal with the regrowth delay in different experimental tumour systems after fractionated X-ray exposures. The data were used to derive $\alpha / \beta$ ratios for the underlying dose dependence of cell survival and, in one study, also to analyse recovery kinetics of sublethal damage. The method of analysis was based on the same postulates as earlier treatments that employed the so-called $F_{\text {e }}$ plots of the inverse of the total dose versus dose per fraction (Douglas and Fowler 1976), but the numerical procedures were different, in being a generalization of the optimization method introduced by Lam et al. (1979). This method, which has found a number of applications (Douglas et al. 1979, Henkelman et al. 1980), offers substantial advantages over the use of the $F_{\mathrm{e}}$ plots. That it is not yet in general use may be due to its requirement of a special, although fairly elementary, computer code and-perhaps primarily - to a certain lack of transparency that is inherent in optimization procedures involving a number of free parameters. In our analysis we have therefore utilized as intermediate step a nonparametric approach which provides an estimate of the $\alpha / \beta$ ratio without the need to estimate further parameters. Although the method has been applied to more complex data (Guttenberger et al. 1990), it will here be explained for the simple case of complete recovery. In the appendix it is shown that the nonparametric method can be 
extended to estimate two parameters in the more complex case of incomplete recovery.

The analysis of regrowth delay is, in its simple form, based on the usual two-fold assumption:

1. Repair of sublethal damage between the individual exposures is complete, and the surviving fractions of the tumour cells for equal absorbed doses, $d$, are equal and follow the same linear-quadratic dependence on dose. The overall surviving fraction, $S$, after a fractionated exposure is then given by the relation:

$$
-\ln S=n\left(\alpha d+\beta d^{2}\right)
$$

or

$$
-\frac{\ln S}{\beta}=\frac{\alpha}{\beta} D+\frac{D^{2}}{n}
$$

where $d$ is the dose per fraction, $n$ is the number of fractions, and $D=n d$ is the total dose; $\alpha / \beta$ is the 'crossover dose', i.e. the dose where the linear component in the survival relation equals the quadratic component.

2. The duration of regrowth, $\tau$, of a tumour to a specified size is related to cell survival and, therefore, implicitly dependent on the same expression, $x$, in dose and number of fractions that determines cell survival:

$$
\tau=\tau(x), \quad \text { with } x=n\left(\alpha / \beta d+d^{2}\right)=\alpha / \beta D+\frac{D^{2}}{n}
$$

The quantity, $x$, which is assumed to be proportional to the logarithm of cell survival and which depends on dose and number of fractions, is termed the 'dose metameter'. The functional dependence between $x$ and the observed effect, $\tau$, is called the 'link function'.

As emphasized in contributions to the problem (Tucker 1984, Thames 1985), it is difficult to assess the validity of the above postulates on the basis of data sets from individual studies. Accordingly such an assessment was not attempted in the preceding articles, and it is also not the subject of this report. The earlier investigations (see Williams et al. 1985) will, instead, be accepted as sufficient evidence that the postulates in Equations (1) and (2) apply to the experimental conditions described in the next section. The subsequent considerations thus deal with the derivation of the $\alpha / \beta$ ratios and of the functional dependence $\tau(x)$ under the assumed validity of the postulates.

For the biological endpoint considered here, i.e. the regrowth delay of a tumour, the functional dependence $\tau(x)$ and the $\alpha / \beta$ ratio are unknown. The familiar method to derive $\alpha / \beta$, the so-called $F_{\mathrm{e}}$ plot of the inverse of the total dose versus dose per fraction of, permits an estimation of $\alpha / \beta$ without the need to assume or determine the underlying dependence, $\tau(x)$. The method is attractively simple, but it suffers from the disadvantage of necessitating somewhat uncertain interpolations between actual data points to obtain isoeffect doses. Accordingly it utilizes only part of the observed data (Taylor and $\mathrm{Kim} 1989$ ). This restriction can substantially reduce the value of the experimental data. A more general procedure is therefore desirable that permits the derivation of the function $\tau(x)$ and the $\alpha / \beta$ ratio by a simultaneous fit to the entire data set for a tumour. The simultaneous fit is, in the method of Lam et al. (1979), obtained by numerical optimization with a postulated analytical expression 
for $\tau(x)$ (Lam et al. use the notation $G$ and $F$ instead of $\tau(x)$ and $x$ ). This is a valid approach, but it is subject to the possible bias that results from the choice of the analytical expression. Such bias can be avoided by a nonparametric method that obviates the need to select an analytical expression for $\tau(x)$. The nonparametric method has the advantage of computational simplicity, and it can either be used by itself or as an intermediate step that provides the necessary guidance for the selection of appropriate functional forms of $\tau(x)$ to be employed in a parametric fit.

\section{Materials and methods}

Three established transplantable tumours of different histology are used to illustrate the method of analysis. The tumour systems, the experimental procedure to transplant the tumour cells, and the irradiation procedures are described elsewhere (Guttenberger et al. 1990). A brief summary of the experimental methods and the conditions of irradiations will therefore be sufficient in the present context.

Tumours were transplanted to $8-10$-week-old inbred female $\mathrm{C} 3 \mathrm{H}$ mice (colony Neuherberg) as $1 \mathrm{~mm}^{3}$ fragments by trocar into the subcutaneous tissue of the right flank under hexobarbital anaesthesia. When they had reached $80-150 \mathrm{mg}$ they were allocated to treatment groups. Six to 12 mice were included in each dose group, and three or four dose levels were used for each fractionation pattern. The tumours were locally irradiated with $300 \mathrm{kV} \mathrm{X}$-rays at a dose rate of $4.6-5 \cdot 7 \mathrm{~Gy} / \mathrm{min}$. The mice were anaesthesized and the tumours were clamped $5 \mathrm{~min}$ prior to and throughout irradiation to ensure local hypoxia. To minimize dose inhomogeneities the mice were placed on a revolving plate $(1 \mathrm{rev} / \mathrm{min})$.

Doses were monitored during irradiation with an ionization chamber placed in one of the exposure positions on the revolving plate. The doses were delivered at intervals of approximately $8 \mathrm{~h}$ in one, two, four or eight equal fractions. Following irradiation the tumour mass was measured regularly once to three times a week, according to the growth rate, until a predetermined size (two to five times the pretreatment size) was reached. The measurements employed a Perspex ruler containing a series of boreholes of increasing diameter, calibrated for tumour mass.

For the analysis the tumour size was normalized to its value at the start of irradiation, and an individual growth curve was plotted for each tumour. The time after treatment to grow to a specified size was estimated from this curve. The absence of a tumour-bed effect was also checked from these individual curves.

\section{Method of analysis}

\subsection{A minimization of 'inversions'}

Figure 1 contains diagrams of the experimental data for the three tumour systems. Each data set consists of $K$ observed regrowth delays, $\tau_{k}(k=1,2, \ldots, K)$, for different combinations, $D_{k}$ and $n_{k}$, of total dose and number of fractions. The regrowth delay, $\tau$, is the difference between the times needed by the exposed and unexposed tumours to reach the specified size. The growth times, $\tau_{0}$, for the unirradiated tumours were taken from previous tumour growth measurements; they are assigned the values $10,9 \cdot 5$, and 6 days for the mammary carcinoma, the Harding Passey melanoma, and the fibrosarcoma. Imprecisions of these values are irrelevant to the nonparametric analysis, and they are not highly critical to the parametric analysis. 

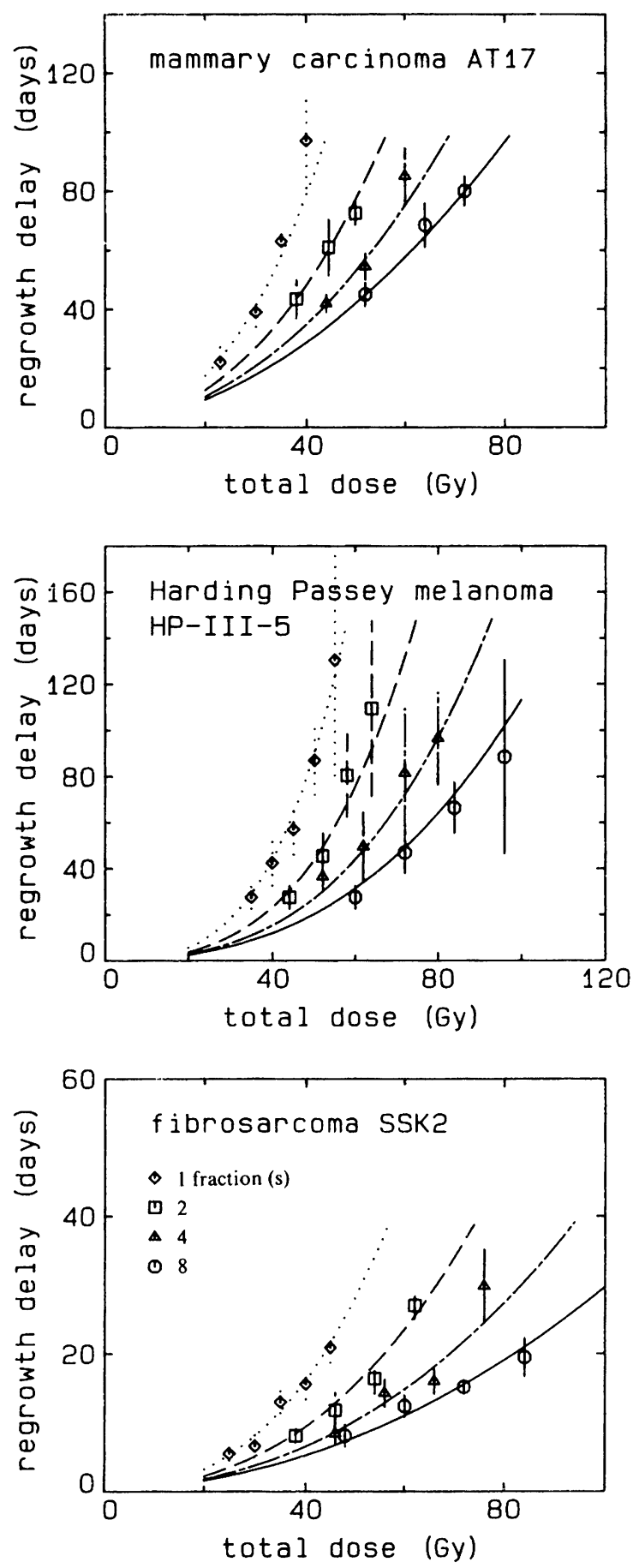

Figure 1. Regrowth delays for three tumour systems. In each experiment four fractionation protocols (one, two, four or eight fractions) were used with equal intervals between fractions $(8 \mathrm{~h})$. The curves result from a fit to the data according to Equations $(6)$ and (2). 

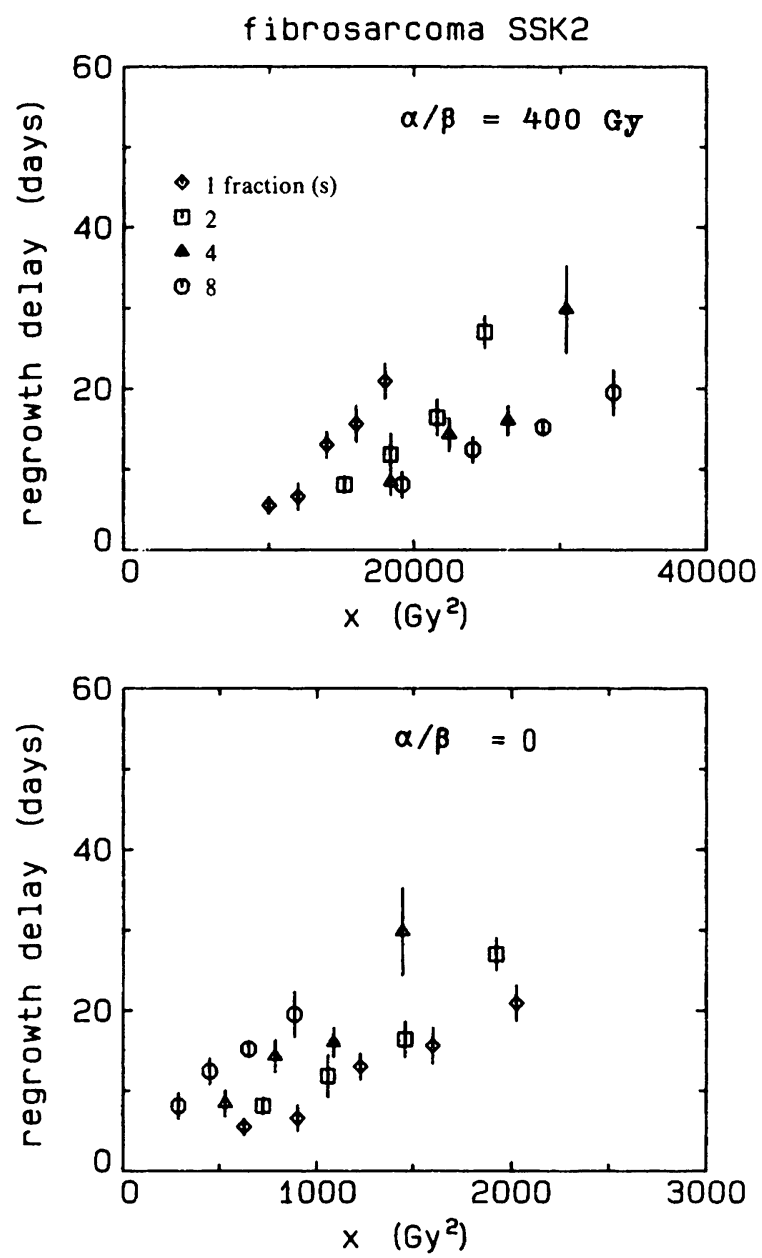

Figure 2. Relation between the observed regrowth delays and the dose metameters for an $\alpha / \beta$ ratio that is too large $(\alpha / \beta=400 \mathrm{~Gy}$, upper panel) or too small $(\alpha / \beta=0$, lower panel).

Assuming a certain value $\alpha / \beta$, one obtains from the experimental data $K$ pairs of metameter values, $x_{k}=\alpha / \beta D_{k}+D_{k}^{2} / n_{k}$, and observed regrowth delays, $\tau_{k}$. If the two basic postulates were fulfilled, and if the assumed $\alpha / \beta$ ratio were equal or nearly equal to the true value, one would obtain a set of points $\left(x_{k}, \tau_{k}\right)$ in the $(x, \tau)$-plane that is close to the actual relationship, $\tau(x)$, between regrowth delay and the metameter $x=\alpha / \beta D+D^{2} / n$. For a wrong value $\alpha / \beta$ one would expect a less consistent dependence, i.e. a larger 'scatter' among the set of points $\left(x_{k}, \tau_{k}\right)$.

As a heuristic step, not as a necessary part of the analysis, one may first consider the $\left(x_{k}, \tau_{k}\right)$ diagrams that result from two wrong assumptions, namely from assumed values of $\alpha / \beta$ that are either clearly too large or too small. The upper panel in Figure 2 gives for the fibrosarcoma SSK2 the diagram for $\alpha / \beta=400 \mathrm{~Gy}$, i.e. for a nearly linear dose dependence; the lower panel gives the diagram for $\alpha / \beta=0$, i.e. for an assumed purely quadratic relation. The upper panel is, apart from the factor $\alpha / \beta$ in 
the abscissa scale, virtually equal to the corresponding diagram in Figure 1 . The salient point in Figure 2 is that both of these badly chosen $\alpha / \beta$ ratios lead to large scatter and are therefore readily recognized as inconsistent with the data. The multifraction data lie too low in the upper panel and too high in the lower panel; the true model must, therefore be in between the two limits, i.e. it must be a linearquadratic expression with some intermediate value $\alpha / \beta$. The more consistent relation obtained with a suitable estimate of $\alpha / \beta$ will be given below (see Figure 4 ).

To derive the correct $\alpha / \beta$, one must search for the value that yields the $\left(x_{k}, \tau_{k}\right)$ diagram with the least degree of scatter. This requires a measure for the extent of the scatter of the points around a common monotone dependence. One possible choice is the Spearman rank correlation coefficient which has been used in the analysis of similar data (Maciejewski et al. 1989, Vegesna et al. 1989). Another simple and meaningful, if somewhat arbitrary, measure is the number, $I$, of 'inversions', i.e. the number of combinations with $x_{k}<x_{j}$ and $\tau_{k}>\tau_{j}$. Such combinations should not occur, except for statistical fluctuations and experimental inaccuracies. In the example of Figure 2, one has 32 inversions for the linear model and 34 inversions for the quadratic model; the number of resulting inversions is considerably smaller for intermediate values of $\alpha / \beta$.

The use of the number of inversions to estimate parameters in generalized linear models has been proposed by Han (1987). As he points out, it is based on the Kendall rank correlation coefficient which is, like the Spearman rank correlation coefficient, a measure of correlation between two variables when their functional dependency is unknown.

The simple counting of inversions would be an acceptable procedure, if the variables $x$ and $\tau$ were measured only on an ordinal scale. But here one can sharpen the analysis by giving suitably chosen weights to the inversions. One weight factor that suggests itself is the square, $\left(\tau_{j}-\tau_{k}\right)^{2} /\left(\sigma_{j}^{2}+\sigma_{k}^{2}\right)$, of the negative difference between the regrowth durations; the denominator in this expression accounts for the standard errors, $\sigma_{j}$ and $\sigma_{k}$, of the observed regrowth delays $\tau_{j}$ and $\tau_{k}$ which are mean values of several measurements. One could also use the data from individual animals, and would then merely use the term $\left(\tau_{j}-\tau_{k}\right)^{2}$ in the weight factor.

An added plausible term in the weight factor is the square, $\left(x_{j}-x_{k}\right)^{2} / \bar{x}$, of the difference between the arguments; it reflects the condition that the (positive) difference $\left(\tau_{k}-\tau_{i}\right)$ between regrowth delays should be largest for large differences $\left(x_{j}-x_{k}\right)$, and that, accordingly, inversions should be less likely for large values of $\left(x_{j}-x_{k}\right)$. The term $\bar{x}$ is the average of all values $x_{k}(k=1, \ldots, K)$; it is included to avoid the artefact, that the weight factors increase with increasing $\alpha / \beta$ ratios merely because the values $x_{k}$ increase. This would bias the $\alpha / \beta$ estimates toward smaller values.

With the above choice of the weight factors one utilizes, instead of the number $I$ of inversions, a sum of weighted squares, $S_{I}$, over the $I$ inversions $(j, k)$, i.e. the combinations with $x_{j}<x_{k}$ and $\tau_{j}>\tau_{k}$ :

$$
S_{I}=\sum_{(j, k)} \frac{\left(\tau_{j}-\tau_{k}\right)^{2}}{\sigma_{j}^{2}+\sigma_{k}^{2}} \frac{\left(x_{j}-x_{k}\right)^{2}}{\bar{x}^{2}}
$$

with:

$$
x_{k}=\frac{\alpha}{\beta} D_{k}+\frac{D_{k}^{2}}{n} \quad \text { and } \quad \bar{x}=\frac{1}{K} \sum_{k=1}^{K} x_{k}
$$


The dependence of the parameter $S_{I}$ on the $\alpha / \beta$ ratio can now serve-without the need to employ an optimization algorithm - to derive estimates of the $\alpha / \beta$ ratios for the different tumour systems. Figure 3 represents in three panels the resulting parameters $S_{I}$. Logarithmic scales of $\alpha / \beta$ and of $S_{I}$ are used to give the dependences over wide ranges from small to large $\alpha / \beta$ ratios.

The minimal values $S_{I}$ result for all tumours at $\alpha / \beta$ ratios close to $30 \mathrm{~Gy}$. These values are in good agreement with $\alpha / \beta$ ratios estimated for these largely hypoxic tumour systems in earlier studies (see Kummermehr et al. 1984). The resulting $\left(x_{k}, \tau_{k}\right)$ diagrams are given in the three panels of Figure 4. There is some remaining degree of scatter, but it lies within the range of standard errors of the observed regrowth delays, and there is now - in contrast to the example in Figure 2-no indication of a systematic trend of the differences between small and large numbers of fractions.

The parameter $S_{I}$ chosen for this analysis is, of course, somewhat arbitrary, and one could select other parameters which might produce further slight improvements in the estimated $\alpha / \beta$ ratios. The choice is, however, justified by the coherent dependences $\tau(x)$ (plotted in Figure 4) for different fraction numbers. The advantage of the approach is its comparative simplicity and transparency, and the absence of a bias arising from the untested assumption of an analytical expression, such as a power function, for the unknown dependence $\tau(x)$.

A disadvantage of the approach is the difficulty to provide standard errors of the estimated ratios $\alpha / \beta$. In the absence of a rigorous expression for the standard error of the parameter $\alpha / \beta$, one uses a nonparametric procedure, the bootstrap method, to estimate the standard errors. The method has been shown (see e.g. Efron 1979, 1982) to be applicable in almost any nonparametric estimation problem and to give, under reasonable conditions, results which are asymptotically correct. The bootstrap method is used here to estimate not only the standard errors but also the bias of the estimated parameters $\alpha / \beta$.

In the actual calculations modified data sets are repeatedly generated, i.e. regrowth-delay values are replicated from normal distributions with means and standard deviations equal to the experimentally observed means, $\tau_{i}$, and the standard deviations, $\sigma_{i}$. For each of the simulated data sets one derives a value $\alpha / \beta$ by the nonparametric estimation procedure, and from a sufficient number of data sets the 'bootstrap distribution' of the parameter $\alpha / \beta$ and 'the bootstrap estimate' of the standard error are obtained. In the computations it was sufficient to generate 100 data sets.

Similar computations have been performed in terms of the simple unweighted number of inversions. The resulting estimates and the standard errors are given in the intermediate panel of Table 1 . One notes that the estimates based on the weighted statistics are more efficient, i.e. that they have smaller standard errors.

The efficiency of the estimate is not the only relevant aspect; one needs to ask also for a possible bias. Han (1987) has shown that his 'maximum rank-correlation estimator' - which is largely analogous to the estimator based on the simple number of inversions - is unbiased under fairly broad conditions. However, these conditions include the requirement that the independent variables, i.e. in the present case the doses and the number of fractions, are random variables with a known distribution. This condition is not met in our data, where the doses and the fraction numbers in the experiments were chosen according to judgement. The bootstrap procedure has therefore been modified to assess the magnitude of a possible bias. 

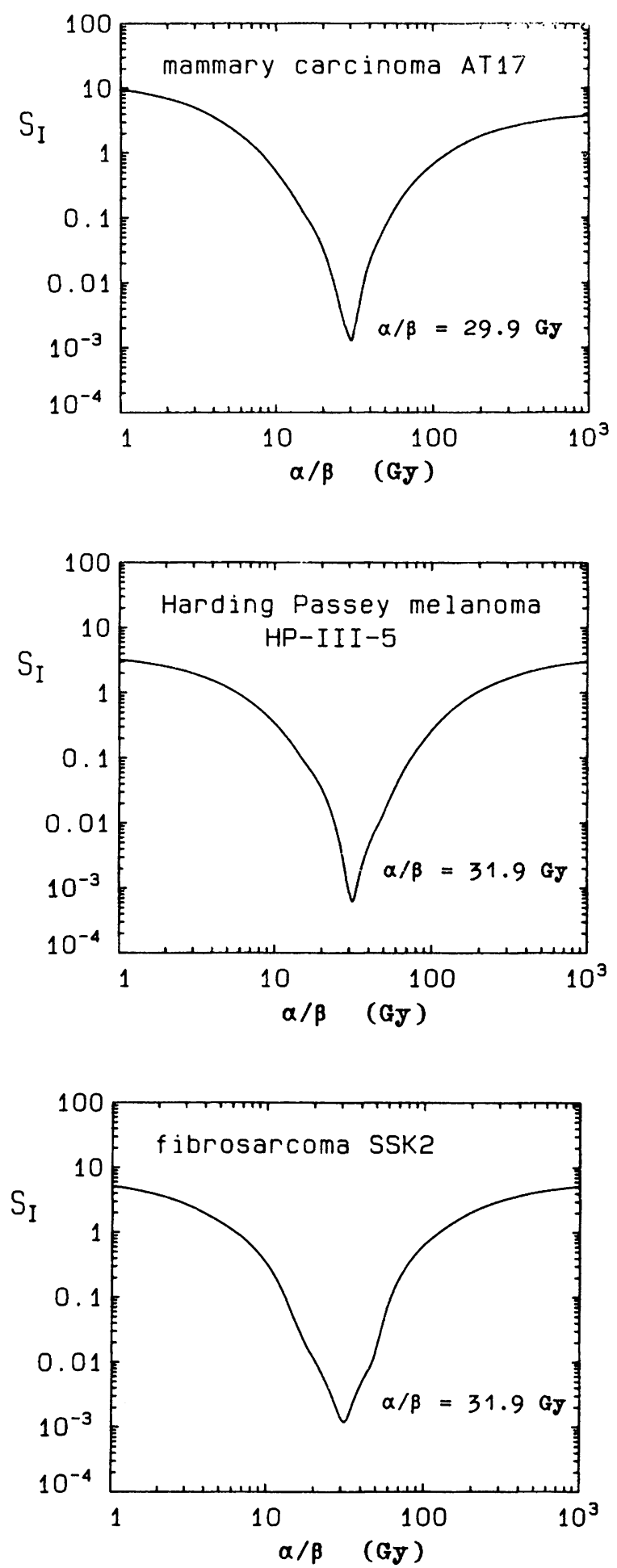

Figure 3. Sum, $S_{I}$, of weighted inversions (see Equation (3)) versus $\alpha / \beta$. The value $\alpha / \beta$ given in each panel corresponds to the minimum of $S_{I}$. 

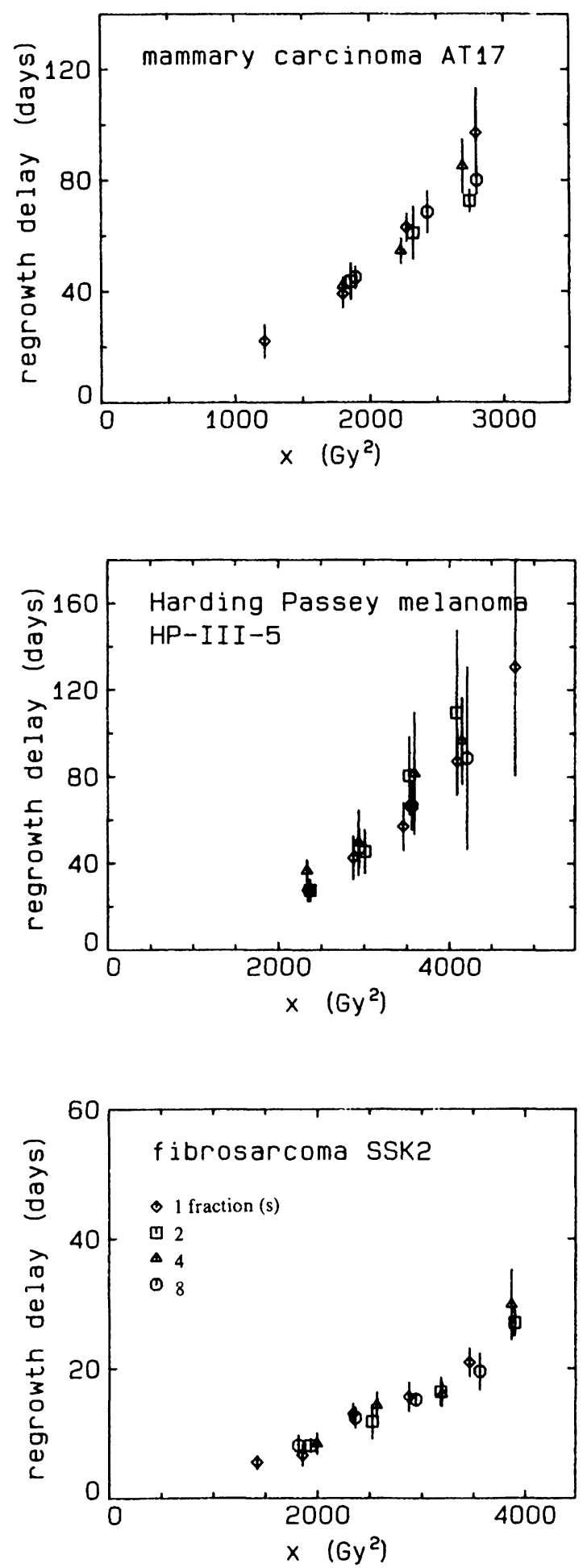

Figure 4. Regrowth delays $\tau_{k}$, versus the dose metameter $x_{k}=\alpha / \beta D_{k}+D_{k}^{2} / n_{k}$. The values of $\alpha / \beta$ are those in Figure 3. 
Table 1. Nonparametric estimates of the $\alpha / \beta$-ratios and standard errors

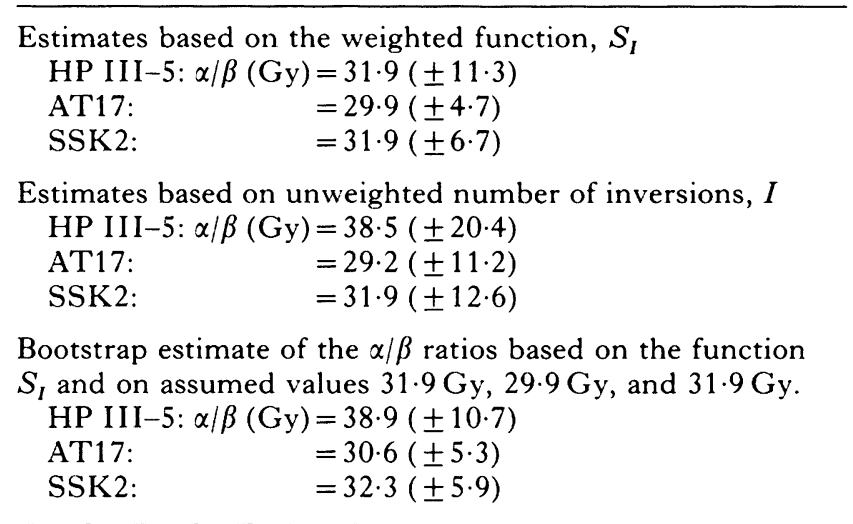

All standard errors are obtained by the bootstrap method.

In the modified procedure the same doses and numbers of fractions, and the same variances of the regrowth delays, are utilized as in the original data, but the mean values of the regrowth delays are replaced by the exact values which result when the relations in Figure 4 are approximated by straight lines. An unbiased estimator should then, on the average, produce the values of $\alpha / \beta$ tabulated in the top panel of Table 1 . In reality one obtains the mean values and the standard errors which are given in the bottom panel of Table 1. One recognizes that there is bias, and that it depends on the characteristics of the data set. As seen in further calculations, the estimated value can be either too small or too large. The magnitudes of the bias are, however, generally smaller than the standard errors. The bias is comparable to the standard error only in the case of the Harding Passey melanoma, but this may merely reflect the fact that these data are, at the higher doses, subject to large statistical errors. One concludes that the nonparametric estimation is biased, but that the bias does not systematically lead to over- or underestimation of the $\alpha / \beta$ ratios, and that it appears to be minor compared to the standard error.

\subsection{Parametric least-squares solution}

The diagrams in Figure 4 suggest fairly simple link functions, $\tau(x)$, and it is therefore of interest to examine parametric fits. Three different models were tentatively employed, to find the least-squares solutions to the data in terms of the relation between the regrowth delay, $\tau$, and the metameter $x=\alpha / \beta D+D^{2} / n$. The three models were:

1. a linear relation

$$
\tau=c x
$$

2. a more general power function

$$
\tau=c x^{p}
$$

3. a linear-quadratic relation

$$
\tau=c_{1} x+c_{2} x^{2}
$$


Relation (5) has two free parameters, $\alpha / \beta$ and $c$; relations (6) and (7) contain three free parameters.

The parameters are estimated from a weighted least-squares fit:

$$
\sum_{k=1}^{K}\left(\tau_{k}-\tau\left(x_{k}\right)\right)^{2} / \sigma_{k}^{2}=\min
$$

$\tilde{\tau}_{k}$ are the observed regrowth delays, and $\tau\left(x_{k}\right)$ are the values from Equations (5)-(7); $\sigma_{k}$ are the standard deviations of the observed values $\tau_{k}$.

Equation (5) is not a linear expression in its two parameters $\alpha / \beta$ and $c$. However, one can use the two parameters $c \alpha / \beta$ and $c$, and then the estimates can be obtained directly using a linear least-squares fitting. For the other equations one needs simple nonlinear optimization algorithms.

A purely linear link function $\tau(x)$ leads, as seen from Table 1 , to fits with excessive sums of squares; it must therefore be rejected. The two other models lead to fits of about equal quality. The choice between the two models is, however, uncritical; the resulting values, $\alpha / \beta$, are, as seen in Table 2 , virtually the same. In Figure 5 the data pairs $\left(x_{k}, \tau_{k}\right)$ are compared to the values $\tau(x)$ resulting from the power-function model. The solid lines in Figure 1 correspond to the same solution, i.e. they represent the regrowth delays according to Equation (6) with the parameters given in Table 2.

\section{Conclusion}

The nonparametric method to analyse the effects of fractionated exposures on tumours or on normal tissues is largely analogous to the method of Lam et al. (1979), but it requires no $a$ priori assumption on the relation between cell depletion and the observed endpoint. The numerical procedure is simple and needs no optimization algorithm. It has here been applied under the familiar assumption of a linear-quadratic dependence for cell survival and provides the estimates of the $\alpha / \beta$ ratios. The method is, however, equally applicable, if other dose dependencies are assumed.

The nonparametric algorithm is particularly suitable for the estimation of a single parameter, but it can also be used for more complex data. The appendix exemplifies this in terms of tumour-regrowth data for exposures with incomplete recovery between fractions.

\section{Appendix: Extension of the nonparametric method}

The nonparametric method can be extended to the more complex situation of exposures with incomplete recovery between fractions.

Guttenberger et al. (1990) have used regrowth-delay data for several tumour systems, to analyse the kinetics of recovery from sublethal damage. They employed the parametric optimization method. We will here exemplify the application of the nonparametric method to the same experimental data.

If there is incomplete recovery between fractions, one obtains, instead of Equation (2), a relation that contains contributions to the quadratic term due to any pair among the $n$ fractions. One usually assumes that the 'sublethal' damage decreases exponentially in time; with an assumed recovery time $t_{0}$ (time for decay of 
Table 2. Estimate of the $\alpha / \beta$ ratios with analytical expressions

(a) Linear model: $\tau=c x$ with $x=\alpha / \beta D+D^{2} / n$

\begin{tabular}{lccccc}
\hline & $\alpha / \beta(\mathrm{Gy})$ & \multicolumn{2}{c}{$c\left(\right.$ day $\left.\mathrm{Gy}^{-2}\right)$} & Sum of squares \\
\hline HP III-5 & $24 \cdot 1$ & $(10 \cdot 7)$ & 0.018 & $(0 \cdot 004)$ & $21 \cdot 3$ \\
AT 17 & 27.5 & $(7 \cdot 4)$ & 0.027 & $(0.005)$ & $17 \cdot 5$ \\
SSK2 & $22 \cdot 7$ & $(7 \cdot 0)$ & 0.0063 & $(0.0011)$ & $28 \cdot 3$ \\
\hline
\end{tabular}

(b) Power model: $\tau=c x^{p}$

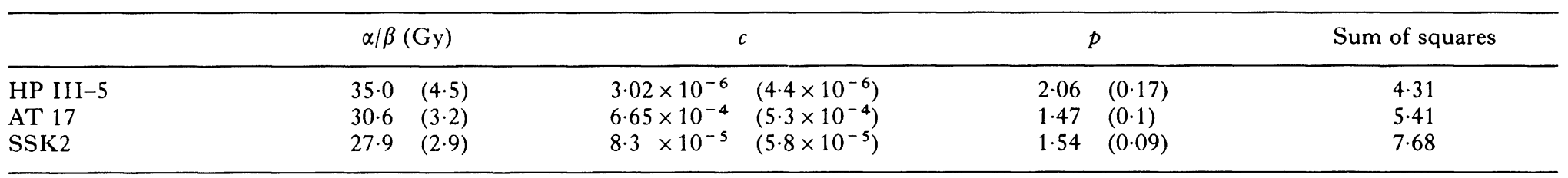

(c) Linear quadratic model: $\tau=c_{1} x+c_{2} x^{2}$

\begin{tabular}{|c|c|c|c|c|c|c|c|}
\hline \multirow[b]{2}{*}{ HP III-5 } & \multicolumn{2}{|c|}{$\alpha / \beta(\mathrm{Gy})$} & \multicolumn{2}{|c|}{$c_{1}\left(\right.$ days $\left.\mathrm{Gy}^{-1}\right)$} & \multicolumn{2}{|c|}{$c_{2}\left(\right.$ days $\mathrm{Gy}^{-2}$ ) } & \multirow{2}{*}{$\frac{\text { Sum of squares }}{4 \cdot 32}$} \\
\hline & $34 \cdot 9$ & $(4 \cdot 1)$ & 0 & $\left(2.6 \times 10^{-3}\right)$ & $5 \cdot 0 \times 10^{-6}$ & $\left(1.0 \times 10^{-6}\right)$ & \\
\hline AT 17 & $30 \cdot 7$ & $(3 \cdot 2)$ & $1.31 \times 10^{-2}$ & $\left(0.28 \times 10^{-2}\right)$ & $5.23 \times 10^{-6}$ & $\left(1.2 \times 10^{-6}\right)$ & $5 \cdot 61$ \\
\hline SSK2 & $28 \cdot 3$ & $(3 \cdot 5)$ & $2.48 \times 10^{-3}$ & $\left(0.6 \times 10^{-3}\right)$ & $1 \cdot 21 \times 10^{-6}$ & $\left(0.22 \times 10^{-6}\right)$ & $7 \cdot 36$ \\
\hline
\end{tabular}

Standard errors are in parentheses. 


\section{power models}
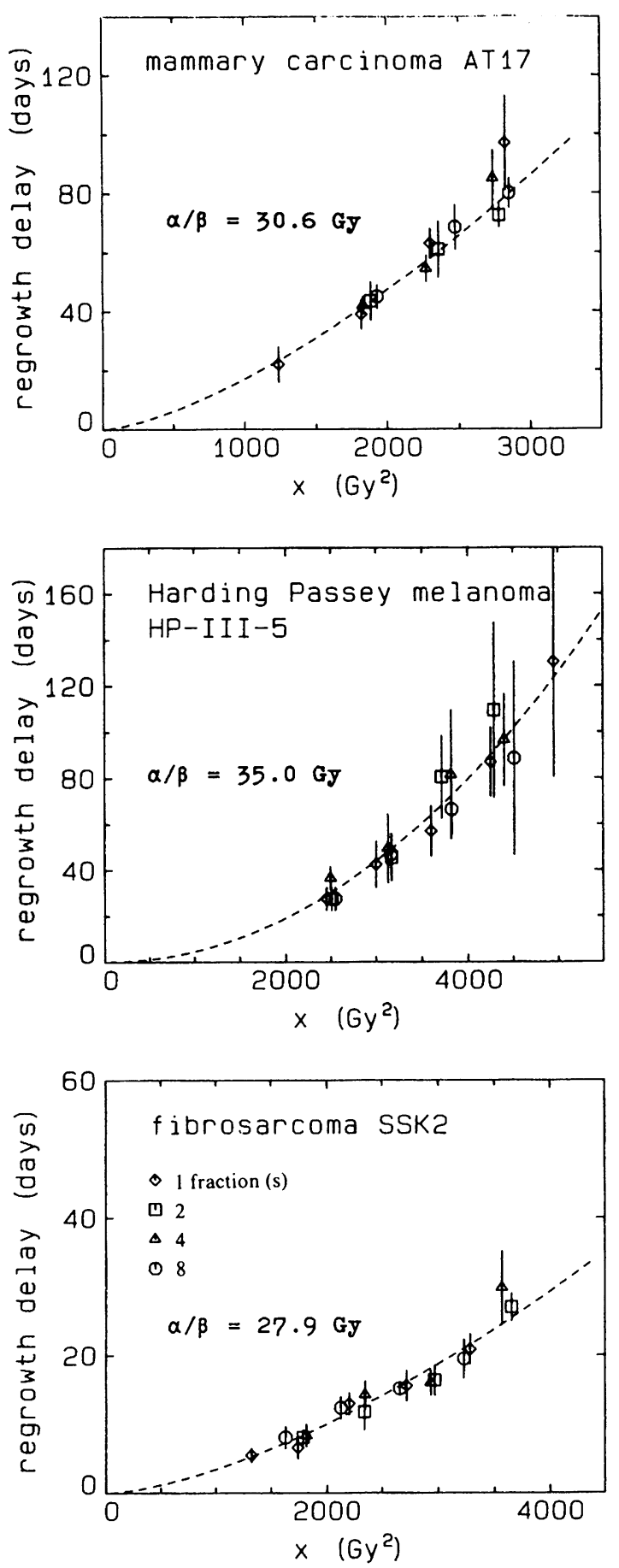

Figure 5. Regrowth delays, $\tau_{k}$, versus the dose metameter $x_{k}=\alpha / \beta D_{k}+D_{k}^{2} / n_{k}$. The values of $\alpha / \beta$ are derived from a least squares fit to Equation (6). 
the damage to decrease to $1 / e$ of its initial value) and with the time interval $\Delta t$ between fractions this leads to the relation:

$$
x=\alpha / \beta D+q_{n}(\Delta t) D^{2} / n
$$

with:

$$
\begin{aligned}
q_{n}(\Delta t) & =\frac{1}{n} \sum_{i, k} \exp \left(-|i-k| \Delta t / t_{0}\right) \\
& =1+\sum_{j=1}^{n-1} \frac{2(n-j)}{n} \exp \left(-j \Delta t / t_{0}\right)
\end{aligned}
$$

$q_{n}(\Delta t)$ equals 1 for $\Delta t \gg t_{0}$, i.e. for complete recovery, and $n$ for $\Delta t=0$, i.e. for no recovery between fractions. It can be shown that this relation agrees with the closed expressions derived by Thames (1985) and also with the formulae utilized by Kellerer and Rossi (1972).

Guttenberger et al. (1990) in their experiments used fractionated exposures with different time intervals of $0,20,55$, and $360 \mathrm{~min}$ between fractions. Their experimental data are given in Figure A1.

Choosing the same statistics, $S_{I}$, that have been described in the main text, but using the changed metameter, one deals with a minimization problem in two dimensions. This can still be handled without an optimization algorithm; one can simply plot isovalue lines of $S_{I}$ in the plane of the two parameters $\alpha i \beta$ and $t_{0}$. The result, for the example of the fibrosarcoma SSK2, is represented in Figure A2. The

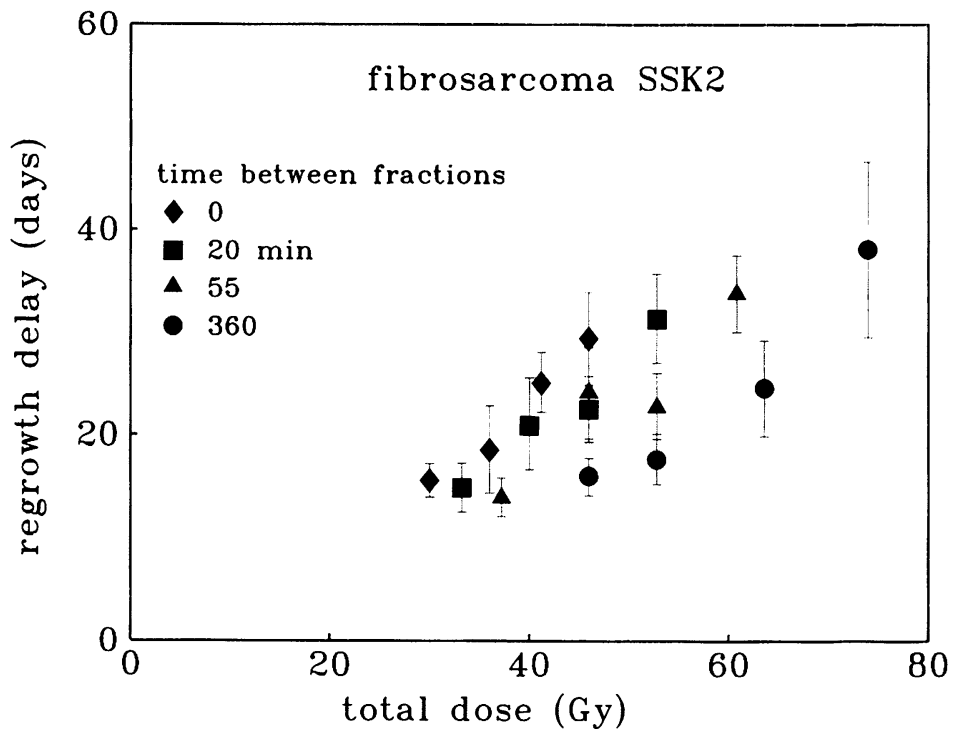

Figure A1. Regrowth delays for the fibrosarcoma SSK2. Four fractions have been used with time intervals between fractions as indicated. 


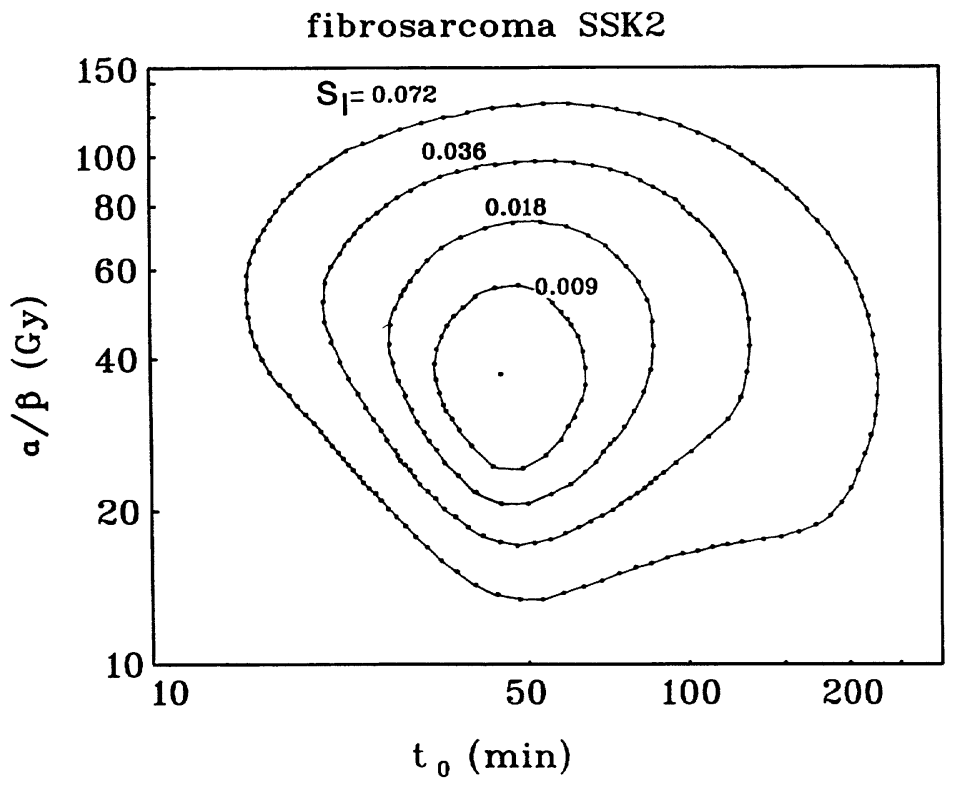

Figure A2. Lines of equal values of the weighted number of inversions, $S_{I}$, in the plane of the parameters $\alpha / \beta$ and $t_{0}$. The point in the centre corresponds to the minimum of $S_{I}$, i.e. to $37 \cdot 3 \mathrm{~Gy}$ and $t_{0}=44 \cdot 6 \mathrm{~min}$.

minimum is obtained for $\alpha / \beta=37.3 \mathrm{~Gy}$ and $t_{0}=44.6 \mathrm{~min}$, which is in good agreement with the values that Guttenberger et al. (1990) have obtained by the parametric method. The regrowth delay as a function of the more complex dose metameter is represented in Figure A3.

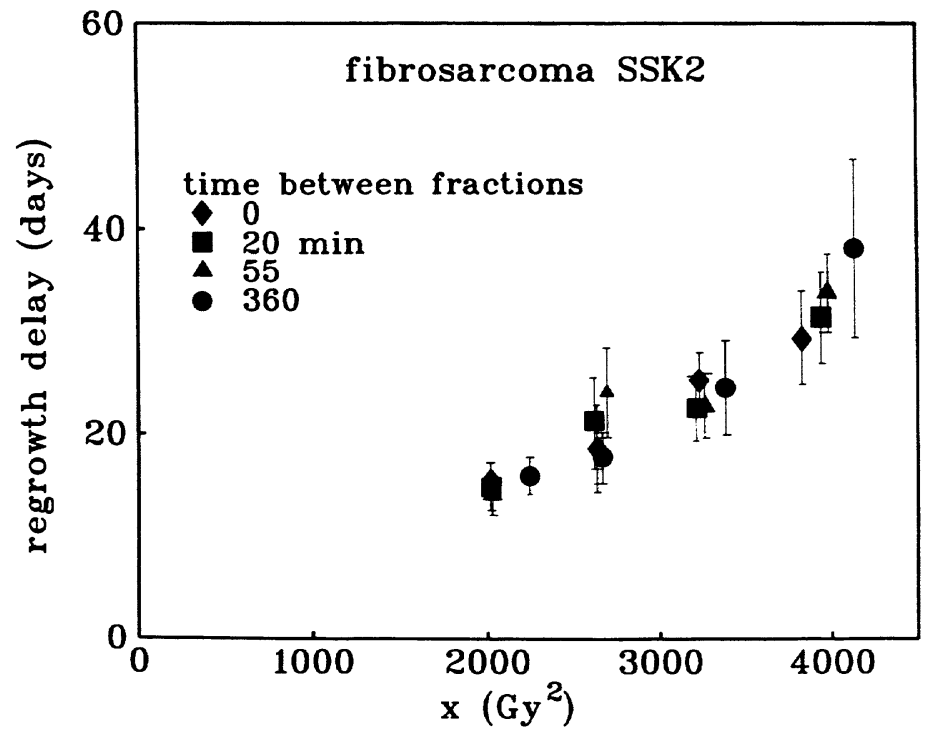

Figure A3. Regrowth delays, $\tau_{k}$, versus the dose metameter $x_{k}=\alpha / \beta D_{k}+q_{n}(\Delta t) \times D_{k}^{2} / n_{k}$ for the values of $\alpha / \beta$ and $t_{0}$ from Figure A2. 
The nonparametric method can, even in this more complex case, serve as a useful intermediate step that provides guidance for the choice of an appropriate analytical expression, $\tau(x)$, in a subsequent parametric analysis. By itself the method is, however, less satisfactory, because it is impracticable to utilize a bootstrap method for the derivation of standard errors; a parametric fit with an optimization algorithm can achieve this more readily.

\section{References}

Douglas, B. G. and Fowler, J. F., 1976, The effect of multiple small doses of X-rays on skin reactions in the mouse and a basic interpretation. Radiation Research, 66, $401-426$.

Douglas, B. G., Henkelman, R. M., Lam, G. K. Y., Föwler, J. F. and Eaves, C. J., 1979, Practical and theoretical considerations in the use of the mouse foot system to derive epithelial stem cell survival parameters. Radiation Research, 77, 453-471.

Efron, B., 1979, Bootstrap methods: another look at the jackknife, Annals of Statistics, 7, 1-26.

Efron, B., 1982, The jackknife, the bootstrap, and other resampling plans. Society for Industrial and Applied Mathematics. CBMS-NSF. Regional conference series in applied mathematics.

Guttenberger, R., Kummermehr, J. and Chmelevsky, D., 1990, Kinetics of recovery from sublethal radiation damage in four murine tumors. Radiotherapy and Oncology, 18, 79-88.

HAN, A. K., 1987, Nonparametric analysis of a generalized regression model: the maximum rank correlation estimator. Journal of Econometrics, 35, 303-316.

Henkelman, R. M., Lam, G. K. Y., Kornelsen, R. O. and Eaves, C. J., 1980, Explanation of dose-rate and split-dose effects on mouse foot reactions using the sametime factor. Radiation Research, 84, 276-289.

Kellerer, A. M. and Rossi, H. H., 1972, The theory of dual radiation action, Current Topics in Radiation Research (Q), 8, 85-158.

Kummermehr, J., Schropp, K. and Neuner, M., 1984, Split dose Erholung in transplantablen Mäusetumoren. Jahresbericht 1983, GSF-Bericht B 1694, pp. $28-34$.

Lam, G. K. Y., Henkelman, R. M., Douglas, B. G. and Eaves, C. J., 1979, Method of analysis to derive cell survival from observation of tissue damage following fractionated irradiation. Radiation Research, 77, 440-452.

Maciejewski, B., Withers, H. R., Taylor, J. and HliniaK, A., 1989, Dose fractionation and regeneration in radiotherapy for cancer of the oral cavity and oropharynx: tumor dose-response and repopulation, International Journal of Radiation Oncology, Biology, Physics, 16, 831-843.

TAYlor, J. M. G. and KIM, D. K., 1989, The poor statistical property of the Fe-plot. International Journal of Radiation Biology, 56, 161-167.

Thames, H. D., 1985, An 'incomplete repair' model for survival after fractionated and continuous irradiations. International Journal of Radiation Biology, 47, 319-339.

TUCKER, S. L., 1984, Tests for the fit of the linear-quadratic model to radiation isoeffect data. International Journal of Radiation Oncology, Biology of Physics, 10, 1933-1939.

Vegesna, V., Withers, H. R. and TAYlor, J. M. G., 1989, Repair kinetics of mouse lung. Radiotherapy and Oncology, 15, 115-123.

Williams, M. V., Denekamp, J. and Fowler, J. F., 1985, A review of $\alpha / \beta$ ratios for experimental tumors: implications for clinical studies of altered fractionation. International Journal of Radiation Oncology, Biology of Physics, 11, 87-96. 